\title{
Enhanced sensitivity to punctate painful stimuli in female patients with chronic low back pain
}

\author{
Christian Puta ${ }^{1,2^{*}}$, Birgit Schulz ${ }^{1}$, Saskia Schoeler ${ }^{3}$, Walter Magerl ${ }^{4}$, Brunhild Gabriel ${ }^{1,3}$, Holger H W Gabriel $^{1}$, \\ Wolfgang $\mathrm{H}$ R Miltner ${ }^{3}$ and Thomas Weiss ${ }^{3}$
}

\begin{abstract}
Background: Chronic low back pain (CLBP) has been shown to be associated with various pathophysiological changes at several level of the sensorimotor system, pointing to a general hypersensitivity in CLBP patients. The aim of the present study was to investigate signs of generalized mechanical pain hypersensitivity in CLBP patients on the hand and on the painful site of the back.

Methods: Pinprick stimulation according to a validated standardized quantitative sensory testing protocol was used in 14 female CLBP patients and 14 healthy controls $(\mathrm{HC})$ matched for sex and age. Stimulus response functions to pinprick stimulation on the skin were examined at the affected back and reference sites (hand palmar and hand dorsum). Data from CLBP patients were compared with $\mathrm{HC}$ and with reference data from the German Research Network on Neuropathic Pain.

Results: We found significant differences in the stimulus response functions between CLBP patients and HC. Pain ratings to the pinpricks were increased for low and moderate pinprick stimuli in CLBP patients. Importantly, this kind of specific pinprick hyperalgesia was found not only for the affected body site (back), but also for the remote reference sites (hand dorsum and hand palmar).

Conclusions: We interpret our results as pointing to changes in the nociceptive processing in CLBP at higher levels of the neuraxis, possibly thalamus and/or attentional control, rather than changes of spinal processing. Alternatively, there might be a higher vulnerability to noxious stimulation in CLBP patients.
\end{abstract}

Keywords: Chronic Low Back Pain (CLBP), Mechanical pain thresholds, Pinprick hyperalgesia, Allodynia

\section{Background}

Chronic low back pain (CLBP) is one of the major health problems in industrialized countries with costs of US\$ 100-200 billions a year [1]. Beyond this economic burden, CLBP patients suffer from reduced quality of life, and loss or limitations of employment. Many also complain of psychopathologies such as depression, anxiety, loss of social activities etc. [2,3]. However, pathophysiological changes in CLBP are still enigmatic and call for additional efforts to demystify the underlying pathophysiology, potentially allowing progress to be made towards successful mechanism-based treatment strategies.

\footnotetext{
* Correspondence: christian.puta@uni-jena.de

${ }^{1}$ Department of Sports Medicine and Health Promotion, Friedrich Schiller University, Wöllnitzer Strasse 42, Jena D-07749, Germany

${ }^{2}$ Center for Interdisciplinary Prevention of Diseases related to Professional

Activities, Friedrich Schiller University, Jena D-07743, Germany

Full list of author information is available at the end of the article
}

CLBP is associated with several pathophysiological changes at various level of the sensorimotor system. At the cortical level, CLBP is associated with functional reorganization in somatosensory and motor regions [4-9]. Changes have also been observed on a behavioural level, namely in attentional control $[10,11]$, during stimulus anticipation [12,13], and in motor behaviour [14-16]. Furthermore, mechanoreceptive [17] and proprioceptive perception is reduced in CLBP [18-20]. However, there are only a few studies using quantitative sensory testing (QST) in CLBP patients. Blumenstiel et al. [21] compared QST profiles of fibromyalgia patients with those of CLBP patients with a focus on fibromyalgia. Investigating QST of the hand and the back, they found significant changes on the backs of CLBP patients with an increased threshold for vibration and a reduced threshold, i.e. hypersensitivity for pressure pain. Their data demonstrate changes to pain

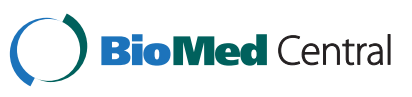


thresholds in CLBP patients that might be interpreted as generalized pain hypersensitivity. These authors did not specifically investigate thresholds at the painful site of CLBP patients but used sites similar to the examination in patients with fibromyalgia. Therefore, they are unable to assess the amount of hypersensitivity with respect to their primary syndrome. In line with the above-mentioned generalized pain hypersensitivity, CLBP patients exhibit lower perception thresholds, lower pain thresholds, lower pain tolerance values, and reduced habituation compared to healthy controls [22-24]. Furthermore, the results of an EEG mapping study indicate enhanced perceptual sensitization and enhanced processing of the sensory discriminative aspect of pain in CLBP patients [25].

The aim of the present study was to investigate signs of generalized mechanical pain hypersensitivity in CLBP patients on the hand and on the painful site at the back. We hypothesized that CLBP patients would exhibit increased sensitivity to noxious punctate mechanical stimuli on both the paraspinal lumbar area and the dorsal and palmar aspects of the hand compared to matched healthy controls.

\section{Methods}

Participants

Fourteen female chronic low back pain (CLBP) patients and fourteen pain-free healthy controls $(\mathrm{HC})$ participated in this study. CLBP patients (for detailed characteristics see Table 1) met the following criteria: 1 . A minimum six month history of low back pain; 2. pain had been classified as 'non-specific low back pain' (no indicators of nerve root problems, e.g. unilateral leg pain, radiating to foot or toes, numbness and/or paraesthesia; straight leg raising test induces leg pain); 3. magnetic resonance imaging (MRI) of the spine showed only age-related changes, but no spinal disorders or disc pathology; 4. no psychiatric disorders and no disease associated with small fibre pathology (e.g.; diabetes mellitus) according to clinical anamnesis. All participants were screened for eligibility by a female clinician (B.S.). B.S. instructed the subjects a week before investigation not to take any analgesic medication for at least 48 hours before the examination. Before examination, subjects confirmed they had not taken any medication during the last 48 hours. All participants were right handed and all were employed. The local ethics committee of the University of Jena approved the whole procedure. Participants gave written informed consent, in compliance with the Declaration of Helsinki.

To evaluate our hypothesis, pain sensitivity to punctate stimuli was tested unilaterally on the most painful body site (paraspinal lumbar; location of measurement: vertebra Th12 to L5) and on two non-painful body sites (hand palmar, hand dorsum; unilaterally on the dominant right

Table 1 Characteristics of the female chronic low back pain (CLBP) patients and female healthy controls (HC)

\begin{tabular}{|c|c|c|c|c|c|c|c|c|}
\hline No. & $\begin{array}{c}\text { Age } \\
\text { (years) }\end{array}$ & $\begin{array}{l}\text { Duration of pain } \\
\text { (month) }\end{array}$ & VAS $^{\text {QST }}$ & NRS $^{4 a v e r a g e}$ & NRS $^{4 m a x}$ & BDI & RDQ & $\begin{array}{l}\text { Reported medications } \\
\text { (as needed) }\end{array}$ \\
\hline 1 & 52 & 360 & 5.8 & 4 & 8 & 11 & 15 & No medication at all \\
\hline 2 & 54 & 168 & 5.1 & 5 & 7 & 12 & 6 & Diclofenac (5-10/month) \\
\hline 3 & 49 & 120 & 1.0 & 2 & 2 & 2 & 2 & Ibuprofen (2-3/month) \\
\hline 4 & 52 & 88 & 3.0 & 3 & 3 & 9 & 5 & Diclofenac (0-8/month) \\
\hline 5 & 49 & 240 & 2.2 & 5 & 8 & 4 & 5 & Ibuprofen (0-1/month) \\
\hline 6 & 54 & 108 & 4.6 & 3 & 6 & 6 & 3 & Diclofenac (2-3/month) \\
\hline 7 & 50 & $>60$ & 3.5 & 5 & 7 & 11 & 5 & Flupirtin (1-10/month) \\
\hline 8 & 56 & $>60$ & 3.5 & 4 & 7 & 9 & 6 & No medication at all \\
\hline 9 & 56 & $>60$ & 1.1 & 4 & 7 & 11 & 2 & Diclofenac (0-10/month) \\
\hline 10 & 44 & $>60$ & 1.0 & 1 & 1 & 7 & 2 & Ibuprofen (0-3/month) \\
\hline 11 & 55 & 147 & 3.6 & 3 & 5 & 4 & 5 & No medication at all \\
\hline 12 & 54 & $>60$ & 3.1 & 5 & 6 & 7 & 3 & Ibuprofen (0-10/month) \\
\hline 13 & 48 & $>60$ & 3.8 & 2 & 4 & 2 & 6 & No medication at all \\
\hline 14 & 56 & $>60$ & 1.0 & 2 & 3 & 11 & 1 & No medication at all \\
\hline CLBP (mean \pm SD) & $52.1 \pm 3.7$ & $>60^{* *}$ & $3.0 \pm 1.7^{* *}$ & $3.4 \pm 1.4^{* *}$ & $5.3 \pm 2.3^{* *}$ & $7.6 \pm 3.5^{* *}$ & $4.7 \pm 3.4^{* *}$ & \\
\hline $\mathrm{HC}($ mean $\pm \mathrm{SD})$ & $51.9 \pm 4.9$ & $0.0 \pm 0.0$ & $0.0 \pm 0.0$ & $0.0 \pm 0.0$ & $0.0 \pm 0.0$ & $1.8 \pm 1.5$ & $0.1 \pm 0.5$ & \\
\hline
\end{tabular}

$\mathrm{VAS}^{\mathrm{QST}}, 100 \mathrm{~mm}$ visual analog scale (VAS): current pain intensity before Quantitative sensory testing (QST); NRS ${ }^{4 a v e r a g e}$, numerical rating scale (NRS) pain intensity rating in response: "How would you rate your average pain over the last four weeks?"; NRS ${ }^{\text {max }}$, numerical rating scale (NRS) pain intensity rating in response: "How would you rate your highest pain intensity experienced within the last four weeks?" VAS/NRS left anchor with $0=$ "no pain" and right anchor with $100 / 10=$ "pain as bad as you can imagine"; BDI - Beck Depression Inventory [28]; RDQ - Roland and Morris Disability Questionnaire [63-65]. Reported medications: Mean use of medication; however, all CLBP patients were without any analgesic medication for at least 48 hours before the examination. All participants were employed. Significant difference between CLBP and HC: ** $-\mathrm{P}<0.001$. 
hand) in CLBP patients. Healthy control subjects were matched on age and gender to the patients (see Table 1). Testing in healthy control subjects was performed at the same regions on the lower back and the dominant hand.

\section{Stimulus response function (SRF) to punctate mechanical stimuli}

In accordance with our hypothesis, we used a test for mechanical pain thresholds from the Quantitative sensory testing (QST) according to the standardized protocol of the German Research Network on Neuropathic Pain (DFNS) [26,27], i.e., the stimulus response function for pinprick stimuli that also allows mechanical pain sensitivity (MPS), another parameter of QST, to be determined. Testing was performed by a DFNStrained investigator.

Stimulus response functions (SRF) to punctate mechanical stimuli were analysed using standard pinprick stimulators (cylindrical tip, $250 \mu \mathrm{m}$ tip diameter) with fixed stimulus intensities that exerted forces of 8, 16, 32, 64, 128, 256, and $512 \mathrm{mN}$ (MRC Systems $\mathrm{GmbH}$, Heidelberg, Germany). All seven pinprick stimuli were applied in a balanced order, five times each at every test site (back paraspinal lumbar, hand palmar, hand dorsum). SRF was assessed as the relationship between the applied forces of the standard pinprick stimulators and the pain rating evoked by each of the pinprick stimulators [28,29], i.e., participants were asked to rate the experienced pain intensity after each stimulation on a verbal rating scale (with 0 indicating "no pain", and 100 indicating "maximal imaginable pain"). To avoid effects of sensitization or fatigue, successive stimuli were not applied at the same spot of skin, but some millimetres away from the preceding stimulation site.

Mechanical pain sensitivity (MPS) was also determined. MPS was assessed as the geometric mean of the pain ratings evoked by each of the seven pinprick stimuli (similar to the standard protocol of DFNS).

In order to exclude a potential influence of skin temperature on the results of testing, skin temperature was assessed for all body sites (back paraspinal lumbar, hand palmar, hand dorsum) before and after the testing. There were no significant skin temperature changes for any of the body sites before vs. after the testing or between patients and controls.

\section{Questionnaires}

Depression was assessed using a German version [30] of the Beck Depression inventory (BDI) [31]. None of the participants suffered from clinically manifest depression (cut-off BDI > 17; Table 1).

Disability was measured using the German version of the 24-item Roland Morris disability questionnaire
(RDQ) [32-34]. RDQ scores range from 0 (no disability) to 24 (maximum disability). Five of the CLBP patients showed clinically-relevant disability $(3<\mathrm{RDQ}$ score $<8)$; 1 patient reported a high level of disability (RDQ $>7$ ); none of the control subjects reported clinically-relevant disability (Table 1).

\section{Data evaluation}

The experienced pain intensities of each of the seven pinpricks (SRF) as well as MPS were normally distributed in log space, and thus were $\log _{10}$-transformed before statistical analysis in accordance with the recommendations of DFNS [27]. For SRF and MPS, a small constant (0.1) was added prior to log-transformation to avoid a loss of values due to zero rating [35]. Data transformed to secondary normal distribution were analysed with analysis of variance (ANOVA) for repeated measurements using the within-subject factors Pinprick (7 intensities from 8 to $512 \mathrm{mN}$ ) and Region (hand dorsum vs. hand palmar vs. lower back) as well as the betweensubject factor Group (CLBP vs. HC). Post-hoc tests were performed using separate ANOVAs. Results were corrected for violations of sphericity using the GreenhouseGeisser approach for epsilon correction of degrees of freedom (when appropriate). All statistical calculations were performed using SPSS 19 Software.

Data were z-transformed to compare the MPS with healthy control data and the data of the DFNS for the hand dorsum using the following expression:

$$
Z-\text { score }=\frac{\left(\text { value }_{\text {individual CLBP patient }}-\text { mean }_{\text {controls }}\right)}{S D_{\text {controls }}}
$$

MPS for each CLBP patient was compared with the group means of healthy controls using z-scores. Z-scores above ' 0 ' indicate a gain of function referring to the higher sensitivity of the CLBP patient to the tested stimuli compared to the healthy controls. Z-scores below ' 0 ' indicate a loss of function when the CLBP patient is less sensitive to the tested stimuli compared with the healthy controls. Z-values below -1.96 or above +1.96 were considered as abnormal for diagnostic purposes (95\% confidence interval $[33,34])$. A $t$-test (two-sided for independent samples) was performed for MPS using the internet-based statistical freeware Simple Interactive Statistical Analysis (SISA; URL: http://www.quantitativeskills. com/sisa/) separately for healthy controls and CLBP patients to compare data with the DFNS reference data as proposed recently [33].

\section{Results}

Detailed analysis of SRF to pinprick stimuli

ANOVA on pain ratings to pinprick stimulation with factors Pinprick, Region, and Group showed significant 
main effects of factors Pinprick $(\mathrm{F}(6,156)=187.02$; $\mathrm{P}<$ $0.001)$, Region $(\mathrm{F}(2,52)=11.57 ; \mathrm{P}<0.001)$, and Group $(\mathrm{F}(1,26)=7.912 ; \mathrm{P}<0.01)$. As expected, the main effect of factor Pinprick resulted from higher ratings to pinpricks with higher force (see Figure $1 \mathrm{~A}-\mathrm{C}$ ). The main effect of factor Region resulted from overall higher pain ratings at the dorsum of the back compared with the hand (hand palmar vs. back: $\mathrm{F}(1,26)=16.62, \mathrm{P}<$ 0.001 ; hand dorsum vs. back: $\mathrm{F}(1,26)=8.38, \mathrm{P}<0.01$; hand palmar vs. hand dorsum: $\mathrm{F}(1,26)=5.99, \mathrm{P}<$ 0.05). The main effect of Group is explained by overall higher pain ratings in the CLBP group as compared to $\mathrm{HC} \quad(\mathrm{F}(1,26)=7.91, \mathrm{P}<0.01$, Cohen's $\mathrm{d}=1.44$; see Figure $1 \mathrm{~A}-\mathrm{C})$. Additionally, there were significant interactions between the factors Pinprick $\mathrm{x}$ Region ( $\mathrm{F}(12$, $312)=16.53, \varepsilon=1.63 ; \mathrm{P}<0.001)$ and Pinprick $\mathrm{x}$ Group $(\mathrm{F}(6,156)=9.07, \varepsilon=3.28 ; \mathrm{P}<0.001)$. One-way ANOVAs for the interaction Pinprick $x$ Group revealed significant differences in pain ratings between groups for the pinpricks $8 \mathrm{mN}, 16 \mathrm{mN}, 32 \mathrm{mN}$ and $64 \mathrm{mN}$ (all $\mathrm{P}<0.05$, corrected for multiple testing; all $\mathrm{d}>1$; range: 1.06 - 1.40). The perceived pain in CLBP patients was 3-5 times higher for low to moderate pinprick forces compared with healthy controls. The difference in pain rating between groups for the $128 \mathrm{mN}$ pinprick was significant at an uncorrected level $(\mathrm{P}<0.037, \mathrm{~d}=0.83)$, whereas no difference in pain ratings between groups was observed for the $256 \mathrm{mN}(\mathrm{P}=0.117, \mathrm{~d}=0.61)$ and $512 \mathrm{mN}$ pinpricks $(\mathrm{P}=0.849 ; \mathrm{d}=0.08)$ (see Figure $1 \mathrm{~A}-\mathrm{C}$ ). No significant interactions other than Pinprick $x$ Group and Pinprick $x$ Region were observed. In particular, there was no 3-way Pinprick $\mathrm{x}$ Region $\mathrm{x}$ Group interaction $(\mathrm{F}(12,312)=1.46, \varepsilon=6.28 ; \mathrm{P}=0.19)$ indicating that the observed differences between groups and the interaction between Pinprick and Group were not different for hand and back.

\section{Detailed analysis of MPS}

ANOVA of MPS revealed main effects of factors Region $(\mathrm{F}(2,52)=11.63, \mathrm{P}<0.001)$ and Group $(\mathrm{F}(1,26)=7.90$, $\mathrm{P}<0.01)$, but no interaction between Region and Group $(F(2,52)=1.93, P=1.55$. As Figure 2 shows, the main effect of factor Region resulted from significantly higher MPS for the back compared with the hand (back vs. hand palmar: $\mathrm{F}(1,26)=16.78, \mathrm{P}<0.001$; back vs. hand dorsum: $\mathrm{F}(1,26)=8.41, \mathrm{P}<0.01$; hand dorsum vs. hand palmar: $\mathrm{F}(1,26)=6.01, \mathrm{P}<0.05)$. The main effect of factor Group is explained by overall higher MPS in the CLBP group as compared to $\mathrm{HC}(\mathrm{F}(1,26)=7.90, \mathrm{P}<0.01$, $\mathrm{d}=1.06$; see Figure 2). MPS of the palmar site of the hand as well as the dorsum of the hand were highly correlated with the MPS at the back (z-score based correlations, hand palmar: $\mathrm{R}^{2}=0.78, \mathrm{P}<0.001$; hand dorsum: $\mathrm{R}^{2}=0.83$, $\mathrm{P}<0.001)$. Furthermore, MPS of the palmar site and of the dorsum of the hand were also highly correlated $\left(\mathrm{R}^{2}=0.81, \mathrm{P}<0.001\right)$.

\section{Incidence of reported pain to punctate stimuli}

Figure 3 shows that the incidence of reported pain increased as a function of stimulus force on the back, hand palmar, and hand dorsum (Figure 3A-C, respectively). The population threshold, i.e., force at which $50 \%$ of pinprick stimuli were reported to be painful, was interpolated as $8 \mathrm{mN}$ for the CLBP patients and $96 \mathrm{mN}$ in healthy controls at the back. Additionally, there were significant differences between groups in the incidence of reported pain on the back for $32 \mathrm{mN}\left(\chi^{2}=12.07\right.$; $\mathrm{p}<$ $0.05)$ and $64 \mathrm{mN}\left(\chi^{2}=10.91 ; \mathrm{p}<0.05\right)$ (see Figure $\left.3 \mathrm{~A}\right)$.

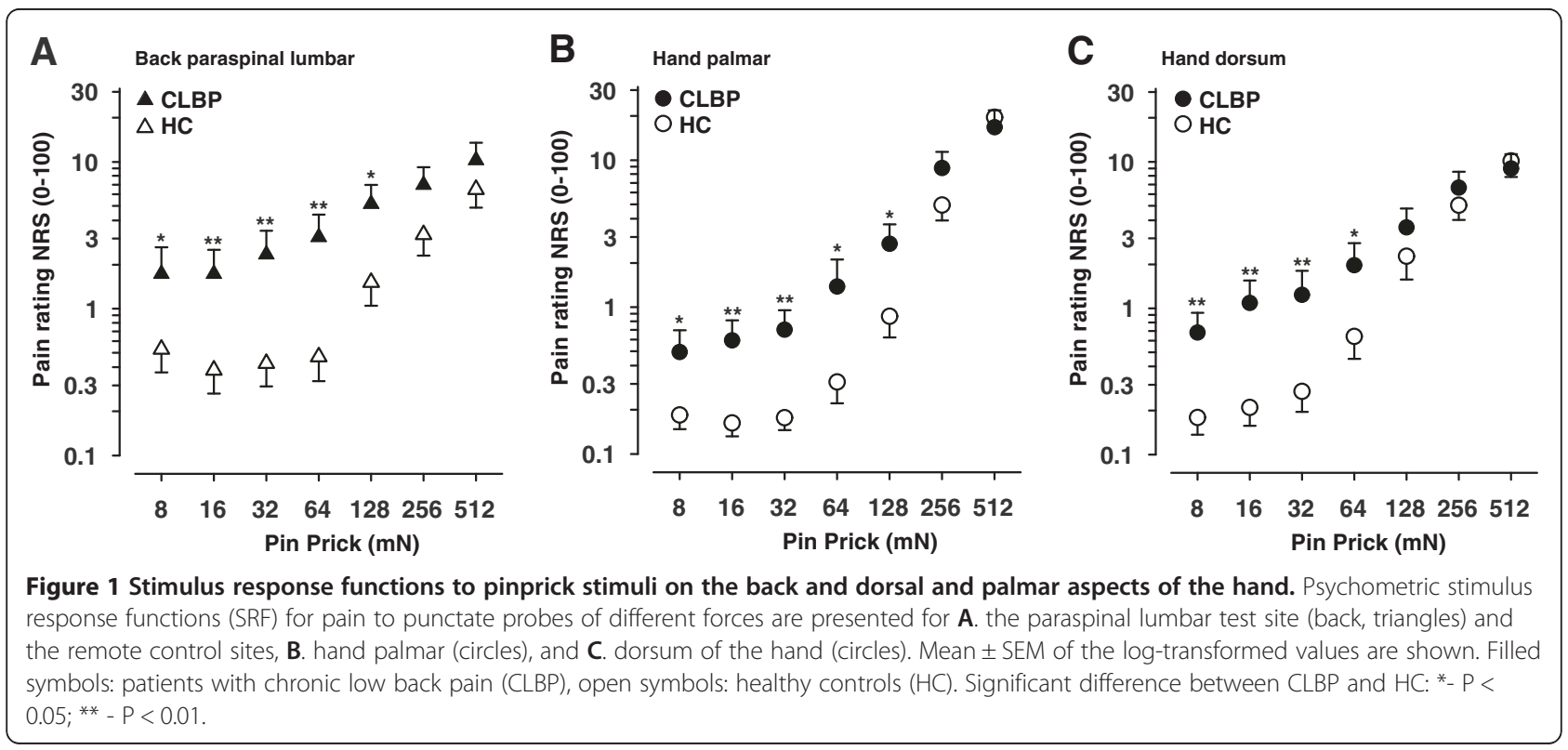




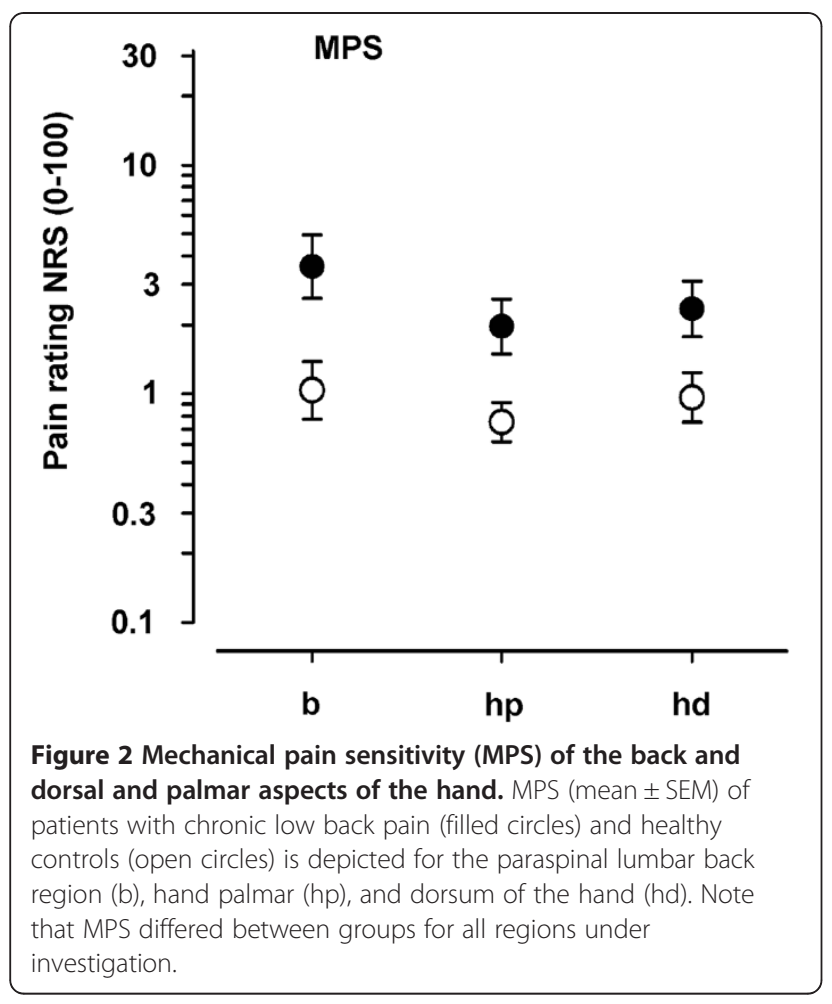

On the hand palmar, the population threshold was also interpolated as $8 \mathrm{mN}$ for the CLBP patients and 96 $\mathrm{mN}$ in healthy controls. There were significant differences between groups in the incidence of reported pain at $32 \mathrm{mN}\left(\chi^{2}=11.03 ; \mathrm{p}<0.05\right)$ and $64 \mathrm{mN}\left(\chi^{2}=\right.$ 11.4; $\mathrm{p}<0.05)$ on the hand palmar (see Figure 3B). For the dorsum of the hand the population threshold was interpolated as $8 \mathrm{mN}$ for the CLBP patients and 48 $\mathrm{mN}$ in healthy controls. There were significant differences between groups in the incidence of reported pain at $8 \mathrm{mN}\left(\chi^{2}=12.92 ; \mathrm{p}<0.05\right), 16 \mathrm{mN}$ $\left(\chi^{2}=12.48 ; \mathrm{p}<0.05\right)$ and $32 \mathrm{mN}\left(\chi^{2}=10.60 ; \mathrm{p}<0.05\right)$ on the hand dorsum (see Figure 3C).

\section{Comparison to reference data}

We also compared our MPS data (detailed data on SRF are not publicly available in the DFNS reference data) with respect to the reference data from the German Research Network on Neuropathic Pain (DFNS). First, we compared the results of our $\mathrm{HC}$ group with the normative data. Results obtained at the hand of our $\mathrm{HC}$ group were in the range of the DFNS reference data as judged by the recommended quality self-control procedure (average zscore of 0.09 for $\mathrm{HC}$ subjects was $<95 \%$ confidence interval of DFNS reference data, recommended to be $<0.25$; [36]) indicating a good agreement with the DFNS standard. The data of our HC group was almost perfectly matched to the reference data $(t=0.05, P=0.62)$. Second, T-tests confirmed a significant group difference for MPS on the hand dorsum for the CLBP patients with respect to our $\mathrm{HC}$ group on the basis of $\mathrm{z}$-transformed values $(\mathrm{t}=$ $2,96 \mathrm{P}<0.05)$ as well as with respect to the reference data $(t=-2.65, P<0.05)$. Furthermore, the MPS $z$-scores for the hand dorsum of CLBP patients normalized to our designated matched control group, and the z-scores normalized to the DFNS reference data [36], were significantly correlated $\left(R^{2}=0.979, P<0.001\right)$ underlining the robustness of results with respect to reference data.

\section{Discussion}

The aim of the present study was to investigate the stimulus response function to punctate mechanical pinprick stimuli in female CLBP patients on the affected
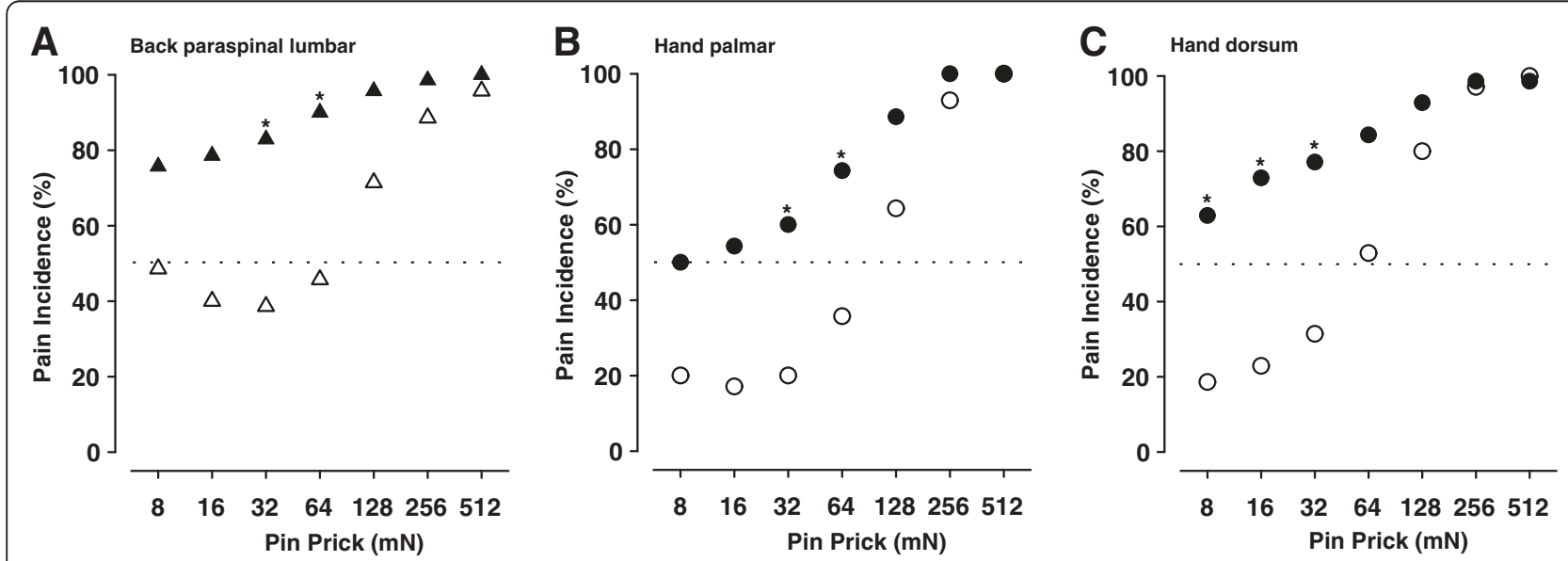

Figure 3 Incidence of the reported pain to pinprick stimuli on the back and palmar and dorsal aspects of the hand. Incidence of reported pain is shown as a function of the pinprick stimulus force $\mathbf{A}$. at the back (triangles), B. hand palmar (circles), and C. the dorsum of the hand (circles). Filled symbols: patients with chronic low back pain (CLBP), open symbols: healthy controls (HC). Population threshold (force at which $50 \%$ of pinprick stimuli were reported to be painful): dotted line in $A, B$ and $\mathbf{D}$. Asterisks in $A, B, C$ depict significant differences $(P<0.05)$ between groups in the incidence of reported pain. 
painful region (lumbar) and at an extraterritorial region (dominant hand). CLBP patients exhibited an enhanced sensitivity and higher pain ratings to punctate mechanical pinprick stimuli, especially pronounced at low to moderate stimulus intensities. Importantly, significantly enhanced pain sensitivity to pinpricks, a hallmark of central sensitization in experimental models and in neuropathic pain, was identified in both the paraspinal lumbar area and the dorsal and palmar surfaces of the hand in CLBP patients compared with matched healthy controls.

\section{Stimulus response function to punctate pinprick stimuli at the back}

Our data revealed significant changes on the back for punctuate mechanical pinprick stimuli, especially pronounced at low to moderate stimulus intensities. SRFs (Figure 1A) showed manifold increases of pain ratings for low to moderate intensities compared to healthy controls. SRFs converged for higher intensities. Compared to healthy controls pain ratings to pinpricks were increased by a factor of 2 to 6 . Beyond the frequent finding of muscle pain thresholds, this is the first report demonstrating hyperalgesia to pinprick stimuli, specifically the pronounced allodynia to low-intensity pinprick stimuli in CLPB patients. These changes are a hallmark of centrally mediated hyperalgesia, and are found in experimental models of central sensitization (neurogenic hyperalgesia [29,36-40] reviewed in $[41,42]$ ) as well as in neuropathic pain patients [43-46]. Specificity for facilitation of this stimulus modality has also been shown in animal models of central sensitization at the level of the spinal dorsal horn, thalamus, and amygdala [47-50].

\section{Stimulus response function to punctate pinprick stimuli on the hand}

Interestingly, we also found significant changes to punctate pinprick stimuli on the hand (MPS, stimulus response function), especially pronounced at low to moderate stimulus intensities (see SRF and pain incidence in Figures $1 \mathrm{~B}-\mathrm{C}, 3 \mathrm{~B}-\mathrm{C}$ ) in CLBP patients. This finding may be of principal importance because it demonstrates changes in pain sensitivity extending far beyond the painful lumbar back. More specifically, there was no significant effect of factor Region suggesting that the increase in sensitivity in CLBP patients to mechanical punctate stimuli is similar for the back and the hand. The mechanisms for this widespread pain sensitivity are largely unknown. Potential explanations include e.g. altered attentional processes, loss of inhibitory pain control, or higher vulnerability in CLBP patients.

A further potential explanation may be related to plasticity at supraspinal levels, for which the thalamus is a prime candidate, since it forms the next relay of the ascending pathway. Receptive field sizes in the thalamus are much larger than in the spinal cord, both in the specific pathways of lateral thalamus projecting to the somatosensory cortices, and in the non-specific pathways of medial thalamus projecting to the anterior cingulate cortex and amygdalae, where receptive field sizes can encompass whole quadrants, or body sides [51]. Several findings in animals support the involvement of the thalamus. In one such study, facial mechanical allodynia spreading from the affected to the contralateral side was accompanied by enhanced thalamic transmission [52]. Contralateral spread of mechanical hyperalgesia and allodynia also appears in more severe cases of migraine in human patients [47,52]. Similar mechanisms have been found in nociceptive plasticity of the spinal or medullary dorsal horn and thalamic plasticity [53,54]. The modality specificity of experimentally-induced mechanical hyperalgesia was also reported as preserved in the amygdala receiving ascending projections from the medial thalamus, as well as descending input from the anterior cingulate cortex and insula [48]. Data on extrasegmental spread however are not yet published. Nevertheless, reverse modulation has been demonstrated in patients with fibromyalgia after two terms of successful local treatment of tender points with widespread relief of signs of hyperalgesia [55].

Alternatively, a relative insufficiency of descending control may passively, or descending facilitation may actively, produce an expansion of receptive field sizes both segmentally and extrasegmentally. Human and animal data have demonstrated that experimentally-induced secondary hyperalgesia involves descending facilitation and spreads rapidly into neighbouring segments $[49,56]$. However, there is no animal data so far demonstrating that sustained spinal nociceptive input may spread sensitization for more than a few segments, e.g. from lumbar to cervical level. Another alternative explanation for the hypersensitivity might be an a priori increased sensitivity to mechanical stimuli in CLBP patients compared to HC. Longitudinal approaches might test this assumption.

\section{Limitations and further directions}

Our sample size of 14 subjects in each group is relatively small, so the study should be extended to larger sample sizes and different centres. Furthermore, we only tested female subjects, whereas previous studies have demonstrated gender differences in pain thresholds (Magerl et al. 2010). Our study should therefore be extended to the investigation of SR functions in male subjects. Additionally, we did not follow the menstrual cycle of our participants although there are known variations in pain perception during the cycle. Nevertheless, in our small 
sample, the sensory changes we report are robust and the effect sizes large (CLBP vs. HC, MPS back: $\mathrm{d}=1.08$; MPS hand dorsum: $\mathrm{d}=0.90$; MPS hand palmar: $\mathrm{d}=1.07$ ).

The data do not allow us to distinguish between the two major hypotheses for this higher-order effect, i.e., sensitization after an aversive event vs. susceptibility. Longitudinal studies might help to solve this question. Alternatively, identifying subjects with a specific pinprick hyperalgesia without CLBP (or other chronic pain symptoms) might be informative about the independence of the observed phenomena.

\section{Conclusions}

In summary, we have found a widespread hypersensitivity to punctate mechanical pinprick stimuli in CLBP patients compared to HC. To our knowledge, this is the first study reporting such a widespread hypersensitivity with a 2 to 6 -fold increase in pain ratings for low to moderate pinprick stimuli in CLBP patients. This kind of specific pinprick hyperalgesia was found not only in the affected painful site (back), but also in the remote reference site on the hand. This result points to higherorder plasticity in CLBP or higher vulnerability rather than to restricted spinal cord mechanisms.

\section{Abbreviations}

CLBP: Chronic Low Back Pain; HC: Healthy Control Subjects; DFNS: German Research Network on Neuropathic Pain; QST: Quantitative Sensory Testing; MPS: Mechanical Pain Sensitivity; SRF: Stimulus Response Function.

\section{Competing interest}

All authors declare no competing of interests.

\section{Authors' contributions}

CP conceived of the study and developed the design, supervised and participated in data acquisition and carried out data analysis, interpretation and drafted the manuscript. BS carried out the acquisition of the subjects, data acquisition, and participated in data analysis. SS carried out data acquisition and participated in data analysis. WM has been involved in interpretation and discussion of data and drafting the manuscript. BG participated in acquisition of the patients and data analysis. HHWG participated in the conception of the study and in drafting the manuscript. WHRM participated in the conception of the study and in drafting the manuscript. TW conceived of the study, participated in the development of its design, coordinated part of the examination, and participated in drafting the manuscript. All authors read and approved the final manuscript.

\section{Funding}

This work was supported by the Federal Ministry of Education and Research BMBF [01EC1003, 01EC1010].

\section{Acknowledgement}

The authors thank Dr. Jeremy Thorne for language advice.

\section{Author details}

'Department of Sports Medicine and Health Promotion, Friedrich Schiller University, Wöllnitzer Strasse 42, Jena D-07749, Germany. ${ }^{2}$ Center for Interdisciplinary Prevention of Diseases related to Professional Activities, Friedrich Schiller University, Jena D-07743, Germany. ${ }^{3}$ Department of Biological and Clinical Psychology, Friedrich Schiller University, Jena D-07743, Germany. ${ }^{4}$ Department of Neurophysiology, Center for Biomedicine and Medical Technology Mannheim (CBTM), Ruprecht Karls University Heidelberg, Mannheim 68167, Germany.
Received: 22 May 2012 Accepted: 19 September 2012

Published: 21 September 2012

\section{References}

1. Balagué F, Mannion AF, Pellisé F, Cedraschi C: Clinical update: low back pain. Lancet 2007, 369(9563):726-728.

2. Carragee EJ: Clinical practice. Persistent low back pain. NEJM 2005, 352(18):1891-1898.

3. Koes BW, van Tulder MW, Thomas S: Diagnosis and treatment of low back pain. BMJ 2006, 332(7555):1430-1434.

4. Wand BM, Parkitny L, O'Connell NE, Luomajoki H, McAuley JH, Thacker M, Moseley GL: Cortical changes in chronic low back pain: current state of the art and implications for clinical practice. Manual Ther 2011, 16(1):15-20.

5. Tagliazucchi E, Balenzuela P, Fraiman D, Chialvo DR: Brain resting state is disrupted in chronic back pain patients. Neurosci Lett 2010, 485(1):26-31.

6. Flor $\mathrm{H}$, Braun $\mathrm{C}$, Elbert $\mathrm{T}$, Birbaumer $\mathrm{N}$ : Extensive reorganization of primary somatosensory cortex in chronic back pain patients. Neurosci Lett 1997, 224(1):5-8.

7. Strutton PH, Theodorou S, Catley M, McGregor AH, Davey NJ: Corticospinal excitability in patients with chronic low back pain. I Spinal Disord Tech 2005, 18(5):420-424.

8. Tsao H, Druitt TR, Schollum TM, Hodges PW: Motor training of the lumbar paraspinal muscles induces immediate changes in motor coordination in patients with recurrent low back pain. J Pain 2010, 11(11):1120-1128.

9. Tsao H, Galea MP, Hodges PW: Driving plasticity in the motor cortex in recurrent low back pain. Eur J Pain 2010, 14(8):832-839.

10. Haggman SP, Sharpe LA, Nicholas MK, Refshauge KM: Attentional biases toward sensory pain words in acute and chronic pain patients. J Pain 2010, 11(11):1136-1145.

11. Grisart J, Plaghki L: Impaired selective attention in chronic pain patients. Eur J Pain 1999, 3(4):325-333.

12. Flor $\mathrm{H}$, Knost $\mathrm{B}$, Birbaumer $\mathrm{N}$ : The role of operant conditioning in chronic pain: an experimental investigation. Pain 2002, 95(1-2):111-118.

13. Moseley GL, Nicholas MK, Hodges PW: Does anticipation of back pain predispose to back trouble? Brain 2004, 127(10):2339-2347.

14. Martel MO, Thibault P, Sullivan MJL: The persistence of pain behaviors in patients with chronic back pain is independent of pain and psychological factors. Pain 2010, 151(2):330-336.

15. MacDonald D, Moseley GL, Hodges PW: Why do some patients keep hurting their back? Evidence of ongoing back muscle dysfunction during remission from recurrent back pain. Pain 2009, 142(3):183-188.

16. MacDonald D, Moseley GL, Hodges PW: People with recurrent low back pain respond differently to trunk loading despite remission from symptoms. Spine 2010, 35(7):818-824.

17. Luomajoki H, Moseley GL: Tactile acuity and lumbopelvic motor control in patients with back pain and healthy controls. Br J Sports Med 2011, 45(5):437-440.

18. Luoto S, Taimela S, Hurri H, Alaranta H: Mechanisms explaining the association between low back trouble and deficits in information processing. A controlled study with follow-up. Spine 1999, 24(3):255-261.

19. Taimela S, Kankaanpää M, Luoto S: The effect of lumbar fatigue on the ability to sense a change in lumbar position. A controlled study. Spine 1999, 24(13):1322-1327.

20. Brumagne $S$, Cordo P, Verschueren S: Proprioceptive weighting changes in persons with low back pain and elderly persons during upright standing. Neurosci Lett 2004, 366(1):63-66.

21. Blumenstiel K, Gerhardt A, Rolke R, Bieber C, Tesarz J, Friederich H-C, Eich W, Treede R-D: Quantitative sensory testing profiles in chronic back pain are distinct from those in fibromyalgia. Clin J Pain 2011, 27(8):682-690.

22. Flor $\mathrm{H}$, Diers $\mathrm{M}$, Birbaumer N: Peripheral and electrocortical responses to painful and non-painful stimulation in chronic pain patients, tension headache patients and healthy controls. Neurosci Lett 2004, 361(1-3):147-150.

23. Kleinbohl D, Holzl R, Moltner A, Rommel C, Weber C, Osswald PM: Psychophysical measures of sensitization to tonic heat discriminate chronic pain patients. Pain 1999, 81(1-2):35-43.

24. Flor $\mathrm{H}$ : Cortical reorganisation and chronic pain: implications for rehabilitation. J Rehabil Med 2003, 35(41 Suppl):66-72.

25. Diers M, Koeppe C, Diesch E, Stolle AM, Holzl R, Schiltenwolf M, van Ackern $\mathrm{K}$, Flor H: Central processing of acute muscle pain in chronic low back 
pain patients: an EEG mapping study. J Clin Neurophysiol 2007, 24(1):76-83.

26. Rolke R, Baron R, Maier C, Tölle TR, Treede R-D, Beyer A, Binder A, Birbaumer N, Birklein F, Bötefür IC, et al: Quantitative sensory testing in the German Research Network on Neuropathic Pain (DFNS): standardized protocol and reference values. Pain 2006, 123(3):231-243.

27. Rolke R, Magerl W, Campbell KA, Schalber C, Caspari S, Birklein F, Treede RD: Quantitative sensory testing: a comprehensive protocol for clinical trials. Eur J Pain 2006, 10(1):77-88.

28. Magerl W, Fuchs PN, Meyer RA, Treede R-D: Roles of capsaicin-insensitive nociceptors in cutaneous pain and secondary hyperalgesia. Brain 2001, 124(9):1754-1764

29. Klein T, Magerl W, Hopf HC, Sandkühler J, Treede R-D: Perceptual correlates of nociceptive long-term potentiation and long-term depression in humans. J Neurosci 2004, 24(4):964-971.

30. Hautzinger M, Bailer M, Worall H: Beck-depressions-inventar (BDI). Bearbeitung der deutschen Ausgabe: Testhandbuch: Bern: Huber; 1994.

31. Beck AT, Ward CH, Mendelson M, Mock J, Erbaugh J: An inventory for measuring depression. Arch Gen Psychiat 1961, 4:561-571.

32. Roland M, Fairbank J: The Roland-Morris Disability Questionnaire and the Oswestry Disability Questionnaire. Spine 2000, 25(24):3115-3124.

33. Roland M, Morris R: A study of the natural history of back pain. Part I: development of a reliable and sensitive measure of disability in lowback pain. Spine 1983, 8(2):141-144.

34. Roland M, Morris R: A study of the natural history of low-back pain. Part II: development of guidelines for trials of treatment in primary care. Spine 1983, 8(2):145-150.

35. Magerl W, Wilk SH, Treede R-D: Secondary hyperalgesia and perceptual wind-up following intradermal injection of capsaicin in humans. Pain 1998, 74(2-3):257-268.

36. Magerl W, Krumova EK, Baron R, Tölle T, Treede R-D, Maier C: Reference data for quantitative sensory testing (QST): refined stratification for age and a novel method for statistical comparison of group data. Pain 2010 151(3):598-605.

37. LaMotte RH, Shain CN, Simone DA, Tsai EF: Neurogenic hyperalgesia: psychophysical studies of underlying mechanisms. J Neurophysiol 1991, 66(1):190-211

38. Ziegler EA, Magerl W, Meyer RA, Treede R-D: Secondary hyperalgesia to punctate mechanical stimuli. Central sensitization to A-fibre nociceptor input. Brain 1999, 122(12):2245-2257.

39. Klein T, Stahn S, Magerl W, Treede R-D: The role of heterosynaptic facilitation in long-term potentiation (LTP) of human pain sensation. Pain 2008, 139(3):507-519.

40. Lang S, Klein T, Magerl W, Treede R-D: Modality-specific sensory changes in humans after the induction of long-term potentiation (LTP) in cutaneous nociceptive pathways. Pain 2007, 128(3):254-263.

41. Magerl W, Klein T: Chapter 33 Experimental human models of neuropathic pain. In Handbook of clinical neurology. 81st edition. Edited by Vinken PJ, Bruyn GW. 2006:503-516

42. Klein T, Magerl W, Rolke R, Treede R-D: Human surrogate models of neuropathic pain. Pain 2005, 115(3):227-233.

43. Baumgärtner U, Magerl W, Klein T, Hopf HC, Treede R-D: Neurogenic hyperalgesia versus painful hypoalgesia: two distinct mechanisms of neuropathic pain. Pain 2002, 96(1-2):141-151.

44. Maier C, Baron R, Tölle TR, Binder A, Birbaumer N, Birklein F, Gierthmühlen J, Flor H, Geber C, Huge V, et al: Quantitative sensory testing in the German Research Network on Neuropathic Pain (DFNS): somatosensory abnormalities in 1236 patients with different neuropathic pain syndromes. Pain 2010, 150(3):439-450.

45. Stiasny-Kolster K, Magerl W, Oertel WH, Möller JC, Treede R-D: Static mechanical hyperalgesia without dynamic tactile allodynia in patients with restless legs syndrome. Brain 2004, 127(4):773-782

46. Fields $\mathrm{HL}$, Rowbotham M, Baron R: Postherpetic neuralgia: irritable nociceptors and deafferentation. Neurobiol Dis 1998, 5(4):209-227.

47. Burstein R, Cutrer MF, Yarnitsky D: The development of cutaneous allodynia during a migraine attack clinical evidence for the sequential recruitment of spinal and supraspinal nociceptive neurons in migraine. Brain 2000, 123(8):1703-1709.

48. Neugebauer V, Li W, Bird GC, Han JS: The amygdala and persistent pain. Neuroscientist 2004, 10(3):221-234
49. Pertovaara A: A neuronal correlate of secondary hyperalgesia in the rat spinal dorsal horn is submodality selective and facilitated by supraspinal influence. Exp Neurol 1998, 149(1):193-202.

50. Simone DA, Sorkin LS, Oh U, Chung JM, Owens C, LaMotte RH, Willis WD: Neurogenic hyperalgesia: central neural correlates in responses of spinothalamic tract neurons. J Neurophysiol 1991, 66(1):228-246.

51. Lenz FA, Weiss N, Ohara S, Lawson C, Greenspan JD: The role of the thalamus in pain. Supp/ Clin Neurophys 2004, 57:50-61.

52. Burstein R, Jakubowski M, Garcia-Nicas E, Kainz V, Bajwa Z, Hargreaves R, Becerra L, Borsook D: Thalamic sensitization transforms localized pain into widespread allodynia. Annals Neurol 2010, 68(1):81-91.

53. Eaton SA, Salt TE: Thalamic NMDA receptors and nociceptive sensory synaptic transmission. Neurosci Lett 1990, 110(3):297-302.

54. Kolhekar R, Murphy S, Gebhart GF: Thalamic NMDA receptors modulate inflammation-produced hyperalgesia in the rat. Pain 1997, 71(1):31-40.

55. Affaitati $G$, Costantini R, Fabrizio A, Lapenna D, Tafuri E, Giamberardino MA: Effects of treatment of peripheral pain generators in fibromyalgia patients. Eur J Pain 2011, 15(1):61-69.

56. Sang CN, Gracely RH, Max MB, Bennett GJ: Capsaicin-evoked mechanical allodynia and hyperalgesia cross nerve territories. Evidence for a central mechanism. Anesthesiology 1996, 85(3):491-496.

doi:10.1186/1471-2377-12-98

Cite this article as: Puta et al:: Enhanced sensitivity to punctate painful stimuli in female patients with chronic low back pain. BMC Neurology 2012 12:98

\section{Submit your next manuscript to BioMed Central and take full advantage of:}

- Convenient online submission

- Thorough peer review

- No space constraints or color figure charges

- Immediate publication on acceptance

- Inclusion in PubMed, CAS, Scopus and Google Scholar

- Research which is freely available for redistribution 